\title{
Thrombus aspiration in primary coronary intervention
}

\author{
Jen-Yu Wang MD, Chuan-Chieh Liu MD, Chih-Cheng Lai MD
}

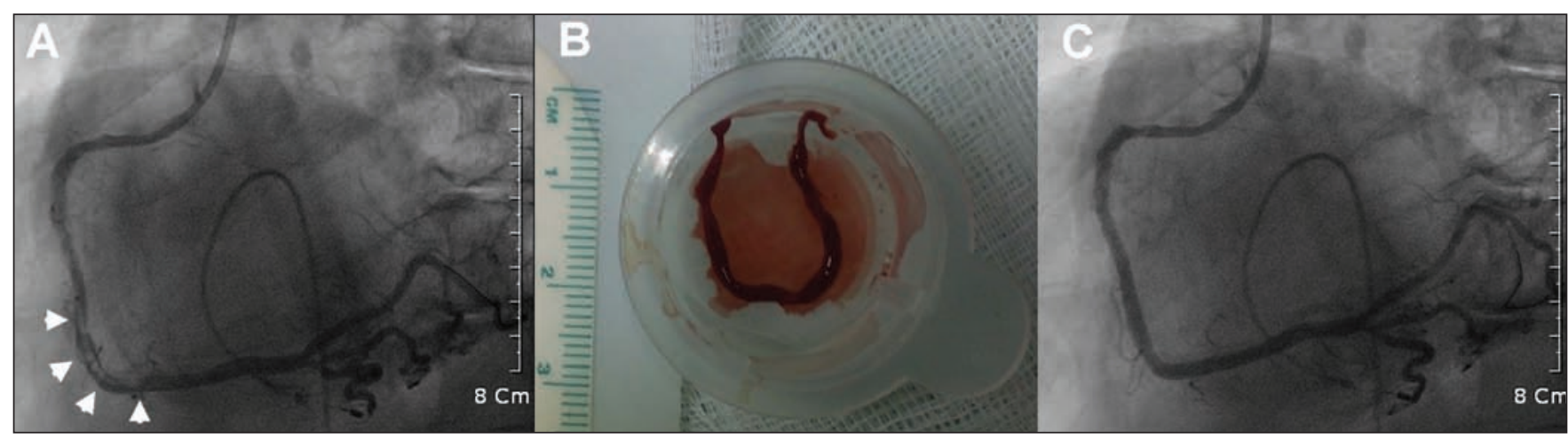

Figure 1 (A) Angiogram, showing occlusion of the right coronary artery with thrombus-containing lesions over the middle portion (arrows). (B) The aspirated thrombus, about $6 \mathrm{~cm}$ in length. (C) Angiogram after coronary angioplasty, showing complete reperfusion.

$\mathrm{A}$ n 84-year-old man presented to the emergency department with severe chest pain that had begun three hours earlier. He was a heavy smoker with a history of hypertension.

On examination, his blood pressure was $120 / 64 \mathrm{~mm} \mathrm{Hg}$, his pulse was regular at 100 beats/min, and his respiratory rate was 20 breaths $/ \mathrm{min}$. The remainder of the physical examination was unremarkable. An electrocardiogram showed a sinus rhythm with ST-segment elevation in leads II, III and aVF. Blood chemistry results showed a creatine kinase (CK) level of 184 (normal 40-290) U/L, a CK MB fraction of 6 (normal < 7) U/L and a troponin I level of 0.32 (normal < $0.5) \mu \mathrm{g} / \mathrm{L}$. Coronary angiography done emergently showed two-vessel disease. The right coronary artery (the infarctrelated vessel) was filled with thrombus-containing lesions over the middle portion (Figure 1A), with evidence of partial reperfusion (Thrombolysis in Myocardial Infarction [TIMI] grade 2 flow). After placement of a guide wire, the QXT extraction catheter (Vascular Solutions, Inc., Minnesota, USA) was advanced into the affected area, and a large red thrombus was aspirated. The thrombus, about $6 \mathrm{~cm}$ in length (Figure 1B), was found to match the filling defect on the angiography. After angioplasty, angiography showed complete reperfusion (TIMI grade 3 flow) (Figure 1C). The patient was discharged from hospital six days later.

From the Department of Internal Medicine, Cardinal Tien Hospital, Fu Jen Catholic University, Taipei, Taiwan

CMAJ 2011. DOI:10.1503/cmaj.092225
The benefit of timely reperfusion by primary coronary intervention has been confirmed in acute ST-segment elevation myocardial infarction. ${ }^{1}$ However, embolization of remaining atherothrombotic debris can lead to microvascular obstruction and worse clinical outcomes. ${ }^{2}$ Several clinical trials have shown that thrombus aspiration can be performed successfully in most patients with acute ST-segment elevation myocardial infarction, and it results in better reperfusion and clinical outcomes than achieved with conventional primary coronary intervention. ${ }^{3,4}$ Recent guidelines support its use in specific circumstances, such as in patients with short ischemic times and large thrombus burden. ${ }^{1}$ Potential adverse events include endothelial damage from forceful aspiration and trauma to tortuous proximal vessels from the aspiration device.

This article has been peer reviewed.

Competing interests: None declared.

\section{REFERENCES}

1. Kushner FG, Hand M, Smith SC Jr, et al. 2009 focused updates: ACC/AHA guidelines for the management of patients with ST-elevation myocardial infarction (updating the 2004 guideline and 2007 focused update) and ACC/AHA/SCAI guidelines on percutaneous coronary intervention (updating the 2005 guideline and 2007 focused update): a report of the American College of Cardiology Foundation/American Heart Association Task Force on Practice Guidelines. J Am Coll Cardiol 2009;54:2205-41.

2. Singh $\mathrm{M}$, Berger $\mathrm{PB}$, Ting $\mathrm{HH}$, et al. Influence of coronary thrombus on outcome of percutaneous coronary angioplasty in the current era (the Mayo Clinic experience). Am J Cardiol 2001;88:1091-6.

3. Silva-Orrego P, Colombo P, Bigi R, et al. Thrombus aspiration before primary angioplasty improves myocardial reperfusion in acute myocardial infarction: the DEAR-MI (Dethrombosis to Enhance Acute Reperfusion in Myocardial Infarction) study. J Am Coll Cardiol 2006;48:1552-9.

4. Svilaas T, Vlaar PJ, van der Horst IC, et al. Thrombus aspiration during primary percutaneous coronary intervention. N Engl J Med 2008;358:557-67. 